Pacific Journal of Mathematics

THE SINGLE VALUED EXTENSION PROPERTY ON A 


\title{
THE SINGLE VALUED EXTENSION PROPERTY ON A BANACH SPACE
}

\author{
JAMES K. FINCH
}

\begin{abstract}
An operator $T$ which maps a Banach space $X$ into itself has the single valued extension property if the only analytic function $f$ which satisfies $(\lambda I-T) f(\lambda)=0$ is $f=0$. Clearly the point spectrum of any operator which does not have the single valued extension property must have nonempty interior. The converse does not hold. However, it is shown below that if $\lambda_{0} I-T$ is semi-Fredholm and $\lambda_{0}$ is an interior point of the point spectrum of $T$, then $T$ does not have the single valued extension property.
\end{abstract}

It will be convenient to use the following definition.

1. Definition. Let $T: X \rightarrow X$ be a closed linear operator mapping a Banach space $X$ into itself, and let $\lambda_{0}$ be a complex number. The operator $T$ has the single valued extension property at $\lambda_{0}$ if $f=0$ is the only solution to $(\lambda I-T) f(\lambda)=0$ that is analytic in a neighborhood of $\lambda_{0}$. Also, $T$ has the single valued extension property if it has this property at every point $\lambda_{0}$ in the complex plane.

2. THEOREM. Let $T$ be a closed linear operator mapping the Banach space $X$ into itself. If $T$ is onto but not one-one, then $T$ does not have the single valued extension property at $\lambda=0$.

Proof. First we produce a candidate for $f$. Choose any $x_{0}$ in $X$ with $\left\|x_{0}\right\|=1$ and $T x_{0}=0$, which is possible since $T$ is not oneone. Since $T$ is a closed operator and is onto, it is an open mapping. The open mapping theorem implies there exists a $k>0$ such that for any element $x \in X$ there is a $y \in X$ with $T y=x$ and $\|y\| \leqq$ $k\|x\|$. Now choose $x_{n}$ inductively so that $T x_{n}=x_{n-1}$ and $\left\|x_{n}\right\| \leqq$ $k\left\|x_{n-1}\right\|$. Define $f(\lambda)=\Sigma x_{n} \lambda^{n}$. Since $\left\|x_{n}\right\| \leqq k^{n}$, the sum converges for $|\lambda|<k^{-1}$, and $f$ is analytic in this neighborhood of zero.

Now to show that $(\lambda I-T) f(\lambda)=0$. Since $T$ is a closed linear operator, so is $\lambda I-T$. Each of the partial sums $\sum_{n=0}^{N} x_{n} \lambda^{n}$ is in the domain of $\lambda I-T$, since each $x_{n}$ was chosen from the domain of T. Furthermore

$$
(\lambda I-T) \sum_{n=0}^{N} x_{n} \lambda^{n}=x_{N} \lambda^{N+1}
$$

and

$$
\left\|x_{N} \lambda^{N+1}\right\| \leqq k^{N}|\lambda|^{N+1} .
$$


But as $N$ goes to infinity, $k^{N}|\lambda|^{N+1}$ converges to zero for $|\lambda|<$ $k^{-1}$. Since $\lambda I-T$ is a closed map, $f(\lambda)=\lim _{N} \sum_{n=0}^{N} x_{n} \lambda^{n}$ is in the domain of $\lambda I-T$ and $(\lambda I-T) f(\lambda)=\lim _{N} x_{N} \lambda^{N+1}=0$.

The function $f(\lambda)$ obtained in the proof of Theorem 2 is certainly not unique. However, it is typical of any function $g$ satisfying $(\lambda I-T) g(\lambda)=0$ in the following sense: Suppose $T$ is any closed operator not having the single valued extension property at $\lambda=0$, and that $g$ is any analytic function defined near $\lambda=0$ satisfying $(\lambda I-T) g(\lambda)=0$. Expand $g$ in a Taylor series around $0: g(\lambda)=$ $\Sigma x_{n} \lambda^{n}$. It can be shown that: (i) each $x_{n}$ is in the domain of $T$; (ii) $T x_{n+1}=x_{n}$ for $n=0,1,2, \cdots$; and (iii) $T x_{0}=0$.

The above discussion holds also at points $\lambda_{0} \neq 0$ if we replace every $T$ by $T-\lambda_{0} I$, and every $\lambda$ by $\lambda-\lambda_{0}$.

There are more interesting ways to express Theorem 2: If $T$ has the single valued extension property, then $T$ is invertible whenever it is onto. Or again, if $T$ has the single valued extension property, then $\lambda_{0}$ is in the spectrum of $T$ if and only if $\lambda_{0}-T$ is not onto. In particular this is true for normal operators, spectral operators, etc.

3. Corollary. Let $T$ be a closed linear operator on a Banach space $X$ and suppose $Y$ is $a \mathrm{cl}$ ) sed invariant subspace. If $T Y=Y$ but $T$ is not one-one on $Y$, then $T$ does not have the single valued extension property at 0.

Actually, in Corollary 3, $Y$ could be a linear manifold that is not closed, provided that it can be given a new norm, larger than the original, for which $Y$ is complete (and hence becomes a Banach space).

4. Corollary. Let $Y$ be the domain of a closed linear operator $S: X \rightarrow Z$, where $Z$ is a Banach space. If $T Y=Y$ but $T$ is not one-one on $Y$ then $T$ does not have the single valued extension property.

5. Corollary. If there is a bounded linear operator on $X$ which is onto but not one-one, then the set of bounded operators that do not have the single valued extension property at 0 has nonempty interior (in the norm topology). And thus the set of operators without the single valued extension property has nonempty interior.

A special case of the following result appears in Colojoarã and Foiaş, Chapter 1. 
6. CoRollary. Let $T$ be a closed linear operator mapping the Banach space $X$ into itself, and assume that the domain of $T$ is dense in $X$ so that the adjoint $T^{*}$ exists. If $T$ is bounded below but is not onto, then $T^{*}$ does not have the single valued extension property. Or alternately, if the range of $T$ is closed and $T$ is one-one but not onto, then $T^{*}$ does not have the single valued extension property.

Proof. If $T$ is bounded below, its range is closed, and $T$ is one-one. Thus the range of $T^{*}$ is the orthogonal set to $\{0\}$, which is all of $X^{*}$; that is, $T^{*}$ is onto. Since $T$ is not onto and the range of $T$ is closed, the null space of $T^{*}$ is not just $\{0\}$. Thus $T^{*}$ is onto but not one-one, and so does not have the single valued extension property by Theorem 2.

A point $\lambda$ is in the limit spectrum of $T$ if and only if there is a sequence $x_{n}$ with $\left\|x_{n}\right\|=1$ and $(\lambda I-T) x_{n}$ converging to 0 .

7. COROllaRy. If the closed linear operator $T$ has the single valued extension property, then the limit spectrum of. $T^{*}$ is the entire spectrum of $T^{*}$. Similarly, if $T^{*}$ has the single valued extension property, then the limit spectrum of $T$ is the entire spectrum of $T$.

A closed linear operator is semi-Fredholm if the range is closed, and the dimension $n(T)$ of the null space or the codimension $d(T)$ of the range is finite (or both). First we investigate the case where the null space is finite dimensional, after a preliminary lemma needed in both proofs.

8. Lemma. Let $T$ be a closed linear operator with closed range mapping a Banach space $X$ into itself, and let $N$ be its null space. For an arbitrary linear manifold $M$ in $X$, if $M+N$ is closed, then the image $T(M)$ of $M$ is closed.

9. THEOREM. Let $T$ be a semi-Fredholm operator mapping a Banach space $X$ into itself with $n(T)$ finite. If the point spectrum of $T$ contains a neighborhood of zero, then $T$ does not have the single valued extension property at $\lambda=0$.

Proof. Let $Y$ be the subset of $X$ given by the intersection of the ranges of $T^{n}$ for all $n$, that is,

$$
Y=\bigcap_{n=1}^{\infty} T^{n} X .
$$


The proof consists of showing that $Y$ is a closed invariant subspace of $X$, that $T$ maps $Y$ onto itself, and that $T$ is not one-one on $Y$. Then, applying Corollary 3 , we see that $T$ does not have the single valued extension property.

It is obvious that $Y$ is a linear manifold in $X$ and that it is invariant under $T$. To show that $Y$ is closed, we need only show that $T^{n} X$ is closed for all $n$. Applying Lemma 9 to $M=T^{n} X$, it follows that $T^{n+1}(X)=T\left(T^{n} X\right)$ is closed if and only if $T^{n} X+N$ is closed. Now by hypothesis $N$ is a finite dimensional closed subspace of $X$, and $T X$ is closed. Since the sum of a closed subspace with a finite dimensional subspace is always closed, $T^{2} X$ is closed. By induction $T^{n} X$ is closed, and hence $Y=\bigcap\left(T^{n} X\right)$ is also closed.

The next step is to show that $T$ maps $Y$ onto itself. Let $R_{n}=T^{n} X$. For any $y$ in $Y$ there is an $x_{n}$ in $R_{n}$ with $T x_{n}=y$. Also, $x_{n}-x_{m}$ is in $N$. Now $R_{n} \cap N$ is a decreasing sequence of subspaces. Since $N$ is finite dimensional, this sequence is eventually constant. That is, for some $m, R_{m} \cap N=R_{k} \cap N$ for all $k \geqq$ $m$. Thus $x_{m}-x_{k}$, which is in $R_{m} \cap N$, is also in $R_{k}$. Since $x_{k}$ was chosen in $R_{k}$, it follows that $x_{m}=x_{k}+\left(x_{m}-x_{k}\right)$ is in $R_{k}$ as well. That is, $x_{m}$ is in $R_{k}$ for all $k \geqq m$; therefore $x_{m}$ is in $Y=\cap R_{k}$. Thus $T$ does map $Y$ onto itself.

It remains to show that the restriction of $T$ to $Y$ is not one-one. It is clear that if $T x=\lambda x$ for some $\lambda \neq 0$, then $x$ is in $Y$. Thus any $\lambda \neq 0$ that is in the point spectrum of $T$ is also in the point spectrum of $T \mid Y$. Hence by our hypothesis, for some $r>0$, every $\lambda$ with $0<|\lambda|<$ $r$ is in the spectrum of $T \mid Y$. Since the spectrum of $T \mid Y$ is closed, it also contains 0 . But then since $T \mid Y$ is onto, it cannot be one-one.

In summary, the resriction of $T$ to $Y$ maps $Y$ onto itself but is not one-one. Hence $T$ does not have the single valued extension property.

Taylor (1966) has shown the following: Let $T$ be an arbitrary linear operator on a vector space (no topological properties are necessary). If $n(T)$ is finite, then $Y=\cap T^{n} X$ satisfies $T Y=$ $Y$. This result can fail if $n(T)$ is infinite.

A slight extension of this theorem is possible. We may replace the assumption that the range of $T$ is closed by: the range of $T^{k}$ is closed for some $k$. Since $n\left(T^{k}\right) \leqq k n(T)$ (see Taylor, 1966), we have that $n\left(T^{k}\right)$ is also finite. Then (using the notation of the proof of Theorem 9) $Y=\cap T^{n} X$ is also equal to $\cap\left(T^{k}\right)^{n} X$. The argument of the proof applied to $T^{k}$ shows that $Y$ is closed and that $T^{k} Y=Y$, but $T^{k}$ is not one-one on $Y$. But of course this means that $T Y=Y$ and $T$ is not one-one on $Y$. Hence, by Corollary 3, $T$ does not have the single valued extension property. 
10. THEOREM. Let $T$ be a closed linear operator mapping a Banach space $X$ into itself, and suppose that the codimension of the range $d(T)$ is finite. If the point spectrum of $T$ contains a neighborhood of zero, then $T$ does not have the single valued extension property at $\lambda=0$.

Note that if $T$ is a closed operator and the codimension (the dimension of $X / R$ ) of the range is finite, then the range is closed and so $T$ is automatically a semi-Fredholm operator. (See Kato, 1966, p. 233, problem 5.7.)

Proof. The idea of this proof is similar to that of Theorem 9. Let $Y=\cap T^{n} X$; it will be shown that $Y$ is a closed subspace invariant under $T$, and that $T$ maps $Y$ onto itself but is not one-one.

First we show that $Y$ is closed; it is obviously a linear manifold that is invariant under $T$. Let $R_{n}=T^{n} X$. Since $d(T)$ is finite, then $d\left(T^{n}\right) \leqq n d(T)$ is also finite. It follows that $R_{n}+N=R_{n} \oplus F_{n}$ where $F_{n}$ is a finite dimensional space. We now show that $R_{n}$ is closed by an induction argument: $R_{n+1}=T\left(R_{n}\right)=T\left(R_{n}+N\right)$. From Lemma 8, $R_{n+1}$ is closed if $R_{n}+N$ is. But $R_{n}+N=R_{n} \oplus F_{n}$ is closed since $R_{n}$ is closed and $F_{n}$ is finite dimensional. Thus $Y=\cap R_{n}$ is closed.

Next it will be shown that $T$ maps $Y$ onto itself. It will be sufficient to show that for some $m, R_{k} \cap N=R_{m} \cap N$ for $k \geqq m$; for then we complete the proof as in Theorem 9. For a proof of the contrapositive, suppose that for an infinite number of $n$ there is a $z_{n}$ in $R_{n} \cap N$ but not in $R_{n+1} \cap N$. Then $z_{n}=T^{n} u_{n}$, where $u_{n}$ is not in $R_{1}$. But the $u_{n}$ are linearly independent. For if $\sum_{k=1}^{K} a_{k} u_{k}=0$, then taking $T^{K}$ and recalling that $z_{k}$ is in $N$, we get $a_{K}=0$. Then recusively we get $a_{k}=0$ for $k=K-1, \cdots, 1$. Thus the $u_{n}$ form an infinite linearly independent set not in $R_{1}$. This contradicts the assumption that the condimension of $R_{1}$ is finite. Thus $T$ maps $Y$ onto itself as in the proof of Theorem 9.

Finally, we show that $T$ is not one-one on $Y$ exactly.as in the proof of Theorem 9.

In conclusion, $Y$ is a closed subspace of $X$ which is invariant under $T$, and $T$ maps $Y$ onto itself but is not one-one on $Y$. Thus $T$ does not have the single valued extension property.

The two theorems above can be summarized to say that: if $T$ is a semi-Fredholm operator and the point spectrum of $T$ contains a neighborhood of zero, then $T$ does not have the single valued extension property at $\lambda=0$. 
The requirement that $T$ be semi-Fredholm in the above theorems seems to be crucial. Even though the range is closed and the point spectrum contains a neighborhood of zero, if $n(T)=d(T)=\infty, T$ may still have the single valued extension property. (Certain normal operators on a nonseparable Hilbert space will work). An attempt to extend the proofs of Theorems 9 and 10 to operators which are not semi-Fredholm must encounter the following two difficulties: The subspace $Y=\cap T^{n} X$ may fail to be closed, or $T$ may not map $Y$ onto itself.

In Theorem 2 it was shown that an operator $T$ which is onto but not one-one does not have the single valued extension property. Such operators are semi-Fredholm operators with $n(T) \geqq 1$ and $d(T)=0 . \quad$ A rather natural extension of this theorem is now possible.

11. Corollary. Let $T$ be a closed linear operator with closed range mapping a Banach space $X$ into itself. If the dimension $n(T)$ of the null space is strictly greater than the codimension $d(T)$ of the range, then $T$ does not have the single valued extension property.

Proof. From the theory of semi-Fredholm operators, for sufficiently small perturbations $S, n(T+S)-d(T+S)=n(T)-d(T)$ (see Kato, Theorem 5.22). Thus for small $\lambda$,

$$
\begin{aligned}
n(\lambda I-T) & =n(T)-d(T)+d(\lambda I-T) \\
& >d(\lambda I-t) \geqq 0 .
\end{aligned}
$$

That is, $\lambda I-T$ is not one-one for $\lambda$ sufficiently small. Thus $T$ is a semi-Fredholm operator with point spectrum containing a neighborhood of zero. By Theorem 10, $T$ does not have the single valued extension property.

One would like to show that if $n(T)<d(T)$, then $T$ does have the single valued extension property (at least at 0 ). Unfortunately, this is not true. For, if $S$ is the right shift on Hilbert space, then $n(S)=0$ and $d(S)=1$; if $T$ is any operator, then $n(T \oplus S)=n(T)$ and $d(T \oplus S)=$ $d(T)+1$. In this way we may extend $T$ to a new operator $T \oplus S \oplus \cdots \oplus S$ with $d$ arbitrarily large and $n$ fixed, without affecting the single valued extendibility (or lack of it).

For a closed linear operator $T$ having a dense domain on a Banach space $X$ there is a unique adjoint operator $T^{*}$ defined on a total subset of the dual space $X^{*}$. 
12. Corollary. Let $T$ be a semi-Fredholm operator on $X$ with domain dense in $X$. If $n(T)<d(T) \leqq \infty$, then $T^{*}$ does not have the single valued extension property at $\lambda=0$.

Proof. For semi-Fredholm operators,

$$
n\left(T^{*}\right)=d(T), \quad \text { and } \quad d\left(T^{*}\right)=n(T) .
$$

Hence $d\left(T^{*}\right)<n\left(T^{*}\right)$, and by Theorem 11, $T^{*}$ does not have the single valued extension property.

13. CoRollary. If $T$ is a closed linear operator on a Banach space with dense domain and closed range, and if both $T$ and $T^{*}$ have the single valued extension property, then $n(T)=d(T)$.

14. CoROllary. Let $T$ be a closed linear operator on a Banach space with dense domain and with $n(T)=d(T)$ finite. Then $T$ has the single valued extension property near $\lambda=0$ if and only if $T^{*}$ does.

Proof. Since $d(T)$ is finite and $T$ is a closed operator, it follows that the range is closed and hence $T$ is a semi-Fredholm operator. If $T$ does not have the single valued extension property near 0 , then $n(\lambda I-T)>0$ for $\lambda$ in a neighborhood of zero. Then

$$
\begin{aligned}
d(\lambda I-T) & =d(T)-n(T)+n(\lambda I-T) \\
& =n(\lambda I-T)>0 .
\end{aligned}
$$

Thus $n\left(\lambda I^{*}-T^{*}\right)=d(\lambda I-T)$ is strictly positive in a neighborhood of zero. But then $T^{*}$ is a Fredholm operator whose point spectrum contains an open set, and so by Theorem $10, T^{*}$ does not have the single valued extension property.

Conversely, suppose $T$ does have the single valued extension property. Then from Theorem $10, n(\lambda I-T)=0$ in a deleted neighborhood of zero (using the fact that $n(\lambda I-T)$ is constant in a deleted neighborhood of a point where $\lambda I-T$ is semi-Fredholm). Hence $d(\lambda I-T)=0$ in this neighborhood. This implies that $T^{*}$ has the single valued extension property near $\lambda=0$.

Two more concepts are useful at this point. Consider the iterates $T^{k}, k=0,1,2, \cdots$, of the operator $T$. The null space $N\left(T^{k+1}\right)$ always contains $N\left(T^{k}\right)$ and may be strictly larger. But if for some 
$p, N\left(T^{p+1}\right)=N\left(T^{p}\right)$, then for all $k>p, N\left(T^{k+1}\right)=N\left(T^{k}\right)$. The smallest $p$ satisfying the above is the ascent of $T$. It may happen that the equation is not satisfied for any $p$; in this case the ascent is infinite. The descent is defined in a similar way with the ranges of $T$ instead of the null spaces. It is the smallest $q$ with $T^{q+1} X=T^{q} X$, and is infinite if no such $q$ exists.

15. Theorem. Let $T$ be a semi-Fredholm operator on $X$. Then $T$ has the single valued extension property near 0 if and only if the ascent of $T$ is finite.

Proof. If the ascent is finite, then certainly $T$ has the single valued extension property near 0 . For if $(\lambda I-T) f(\lambda)=0$ and $f(\lambda)=\Sigma x_{n} \lambda^{n}$, then $x_{n}$ is in $N\left(T^{n+1}\right)$ but not in $N\left(T^{n}\right)$. Hence $N\left(T^{n+1}\right) \neq N\left(T^{n}\right)$ for any $n$.

Suppose that the ascent is infinite. Since $T$ is semi-Fredholm, if the nullity $n(T)$ is infinite, then the deficiency $d(T)$ is finite; and, by Theorem 11, $T$ does not have the single valued extension property. Thus assume that $n(T)$ is finite, and let

$$
Y=\bigcap_{n=1}^{\infty} T^{n} X
$$

As was shown in the proof of Theorem $9, Y$ is a closed, invariant subspace, and $T$ maps $Y$ onto $Y$. Since $n(T)<\infty$, the null space $N$ in finite dimensional, and so $\left(T^{k} X\right) \cap N$ is eventually constant. Since the ascent is infinite, $\left(T^{k} X\right) \cap N \neq(0)$, for all $k$. It then follows that $Y \cap N \neq(0)$; that is, $T$ is not one-one on $Y$. From Corollary 3, $T$ does not have the single valued extension property.

16. COROllary. If $T$ is a semi-Fredholm operator with domain dense in $X$, then $T^{*}$ has the single valued extension property if and only if the descent of $T$ is finite.

Proof. Since the range of $T^{k}$ is closed for all $k$ (as was shown in the proofs of Theorems 9 and 10), the null space of $T^{*^{k}}$ is the set of $x^{*}$ orthogonal to the range of $T^{k}$. Hence the ascent of $T^{*}$ is the descent of $T$, and the conclusion follows by Theorem 15 .

Acknowledgement. This paper is based on the author's Ph.D. dissertation at the University of Illinois. 


\section{REFERENCES}

1. Errett Bishop, A duality theory for arbitrary operators, Pacific J. Math., 9 (1959), 379-397.

2. Ion Colojoară and Ciprian Foias, Theory of Generalized Spectral Operators, New York: Gordon and Breach, 1968.

3. Nelson Dunford, Spectral operators, Pacific J. Math., 4 (1954), 321-354.

4. Nelson Dunford and Jacob Schwartz, Linear Operators, New York: Interscience 1957.

5. Seymour Goldberg, Unbounded Linear Operators; Theory and Applications, New York: McGraw Hill, 1966.

6. Tosio Kato, Perturbation Theory for Linear Operators, New York: Springer-Verlag, 1966.

7. G. M. Keselman, On the single-value analytic continubility of the resolvent of a bounded linear operator, (Russian), Uspekhi Matematicheskikh Nauk, 17 (1962), 135-139.

8. D. G. Lay, Spectral analysis using ascent, descent, nullity, and defect, Mathematische Annalen, 184 (1967), 197-214.

9. Angus E. Taylor, Theorems on ascent, descent, nullity, and defect of linear operators, Mathematische Annalen, 163 (1966), 18-49.

10. F. H. Vasilescu, Residual properties for closed operators on Fréchet spaces, Illinois J. Math., 15 (1971), 377-386.

Received July 17, 1974.

UNIVERSITY OF SAN FRANCISCO 



\section{CONTENTS}

Zvi Artstein and John A. Burns, Integration of compact set-valued functions

J. A. Beachy and W. D. Blair, Rings whose faithful left ideals are cofaithful

Mark Benard, Characters and Schur indices of the unitary reflection group $[321]^{3}$

H. L. Bentley and B. J. Taylor, Wallman rings ............................. 15

E. Berman, Matrix rings over polynomial identity rings II ............... 37

Simeon M. Berman, A new characterization of characteristic functions of absolutely continuous distributions ........................ 323

Monte B. Boisen, Jr. and Philip B. Sheldon, Pre-Prüfer rings ............ 331

A. K. Boyle and K. R. Goodearl, Rings over which certain modules are injective

J. L. Brenner, R. M. Crabwell and J. Riddell, Covering theorems for finite nonabelian simple groups. $V$

H. H. Brungs, Three questions on duo rings .................................... 345

Iracema M. Bund, Birnbaum-Orlicz spaces of functions on groups ....351

John D. Elwin and Donald R. Short, Branched immersions between 2-manifolds of higher topological type

J. K. Finch, The single valued extension property on a Banach space ................................................................................. 61

J. R. Fisher, A Goldie theorem for differentiably prime rings ............ 71

Eric M. Friedlander, Extension functions for rank 2, torsion free abelian groups ...................................................................... 371

J. Froemke and R. Quackenbusch, The spectrum of an equational class of groupoids

B. J. Gardner, Radicals of supplementary semilattice sums of

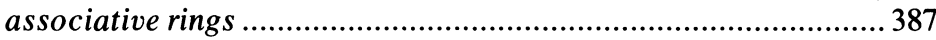

Shmuel Glasner, Relatively invariant measures ...............................393

G. R. Gordh, Jr. and Sibe Mardešić, Characterizing local connectedness in inverse limits...

S. Graf, On the existence of strong liftings in second countable topological spaces

S. Gudder and D. Strawther, Orthogonally additive and orthogonally increasing functions on vector spaces ........................................427

F. Hansen, On one-sided prime ideals .......................................... 79

D. J. Hartfiel and C. J. Maxson, A characterization of the maximal monoids and maximal groups in $\beta x$.

Robert E. Hartwig and S. Brent Morris, The universal flip matrix and the generalized faro-shuffle 


\section{Pacific Journal of Mathematics}

Vol. 58, No. 1

March, 1975

John Allen Beachy and William David Blair, Rings whose faithful left ideals are cofaithful .................................... 1

Herschel Lamar Bentley and Barbara June Taylor, Wallman rings ........ 15

Elizabeth Berman, Matrix rings over polynomial identity rings. II ...... 37

Ann K. Boyle and Kenneth R. Goodearl, Rings over which certain modules are injective ................................. 43

J. L. Brenner, Robert Myrl Cranwell and James Riddell, Covering theorems for finite nonabelian simple groups. $V \ldots \ldots \ldots \ldots \ldots \ldots \ldots \ldots \ldots$

James Kenneth Finch, The single valued extension property on a Banach

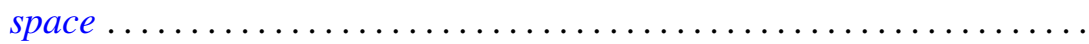

John Robert Fisher, A Goldie theorem for differentiably prime rings........ Friedhelm Hansen, On one-sided prime ideals .................... Jon Craig Helton, Product integrals and the solution of integral equations..........................................

Barry E. Johnson and James Patrick Williams, The range of a normal

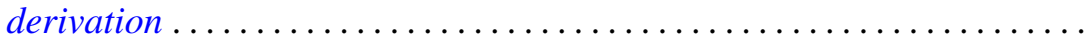

Kurt Kreith, A dynamical criterion for conjugate points ............ 123

Robert Allen McCoy, Baire spaces and hyperspaces .............. 133

John McDonald, Isometries of the disk algebra ................ 143

H. Minc, Doubly stochastic matrices with minimal permanents ......... 155

Shahbaz Noorvash, Covering the vertices of a graph by vertex-disjoint paths. ...

Theodore Windle Palmer, Jordan *-homomorphisms between reduced Banach*-algebras

Donald Steven Passman, On the semisimplicity of group rings of some

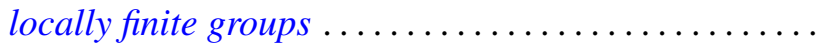

Mario Petrich, Varieties of orthodox bands of groups .

Robert Horace Redfield, The generalized interval topology on distributive

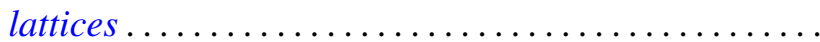

James Wilson Stepp, Algebraic maximal semilattices .... . .

Patrick Noble Stewart, A sheaf theoretic representation of rings with Boolean orthogonalities ........................

Ting-On To and Kai Wing Yip, A generalized Jensen's inequality......... 255

Arnold Lewis Villone, Second order differential operators with self-adjoint

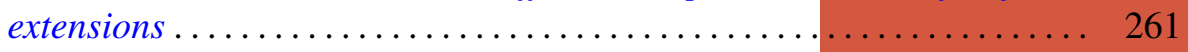

Martin E. Walter, On the structure of the Fourier-Stieltjes algebra ....... 267

John Wermer, Subharmonicity and hulls .................... 283

Edythe Parker Woodruff, A map of $E^{3}$ onto $E^{3}$ taking no disk onto a

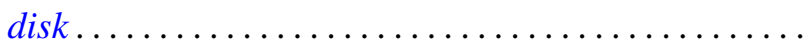

